\title{
ENERGIA SOLAR E SUA VIABILIZAÇÃO
}

\author{
André Emiliano de Barros Nardes \\ George Hallan Marques dos Santos \\ Letícia Estefani Sousa Santos \\ Mariana de Castro Marques \\ Rafaela Aguiar Araújo \\ Ronilson Jardim Sousa
}

RESUMO: Os painéis fotovoltaicos possibilitam a maneira mais inteligente de se converter a energia solar diretamente em energia elétrica, quando a luz solar atinge uma célula fotovoltaica, é formada uma corrente elétrica pequena, sendo assim recolhida e injetada para os outros componentes que formam o sistema. Este trabalho visa o uso da energia sustentável junto a energia elétrica que quando combinada contribui para redução de custo e com isso a valorização de empreendimentos e residências, utilizando telhas fotovoltaicas e painéis fotovoltaicos em quintais de residências e além de estudar a viabilidade do uso de materiais recicláveis, ou RCD para fabricação de telhas para maior economia, analisar o alto consumo de energia elétrica e a diminuição do nível dos rios, comparando investimento e visando melhorar o custobenefício. Um sistema elétrico de potência abrange três principais partes, sendo elas a geração, transmissão e distribuição de energia elétrica. Além de que cerca da metade do consumo de energia no território nacional acontece em edificações residenciais ou comerciais, portanto, o desenvolvimento de telhas solares é uma melhoria de aproveitamento da radiação solar no Brasil, podendo assim adequar nessas edificações onde temos maiores consumo de energia. Essas telhas solares também possuem produção localmente no Brasil sendo mais acessível para compra e com isso diminuir no consumo de energia tradicional de forma ecológica.

Palavras-chave: Energia elétrica. Energia solar. Telhas fotovoltaicas. Sistema de energia. Painéis fotovoltaicos.

Abstract: Photovoltaic panels enable the smartest way to convert solar energy directly into electrical energy, when sunlight hits a photovoltaic cell, a small electric current is formed, thus being collected and injected to the other components that form the system. This work aims at the use of the sustainable energy together with the electric energy that when combined contributes for cost reduction and with this the valuation of enterprises and residences, using photovoltaic roof tiles and photovoltaic panels in backyards of residences and besides studying the viability of the use of recyclable materials, or RCD for manufacture of roof tiles for larger economy, to analyze the high consumption of electric energy and the decrease of the level of the rivers, comparing investment and aiming at improving the cost-benefit. A power electrical system comprises three main parts, being the generation, transmission and distribution of electrical energy. In addition to the fact that about half of the energy consumption in the national territory happens in residential or commercial buildings, therefore, the development of solar roof tiles is an improvement in the use of solar radiation in Brazil, thus being able to adapt 
these buildings where we have higher energy consumption. These solar tiles are also produced locally in Brazil, making them more accessible for purchase and thus reducing traditional energy consumption in an ecological way.

Keywords: Electricity. Solar energy. Photovoltaic tiles. Energy system. Photovoltaic panels.

\section{INTRODUÇÃO}

A construção civil expande-se e evolui cotidianamente, sempre apresentando técnicas a fim de facilitar o desenvolvimento sustentável de um projeto. A capacitação da mão de obra e o uso de materiais adequados é parte essencial dentro de um projeto, visto que em diversos canteiros de obras ainda há falhas por falta de capacitação e mal-uso de materiais determinados para inúmeros serviços. É visível a preocupação dos profissionais em tornar seus projetos mais sustentáveis, buscando atender normas e valorizar novos empreendimentos. Com isso um ponto importante a ser observado é o investimento em energia fotovoltaica, que é considerada energia limpa, pois não agride o meio ambiente e pode substituir a energia elétrica utilizada atualmente.

Segundo a ONS (Operador Nacional do Sistema Elétrico) a energia fotovoltaica é a energia do amanhã pois através dela é possível abastecer uma residência, um comercio, uma indústria sem agredir o meio ambiente, o órgão ressalta que investir nesse ramo hoje é pensar num amanhã sustentável.

Empresas especializadas na fabricação e instalação de telhados e placas fotovoltaicas, como por exemplo a Vegasolar, garantem que é um projeto de custo-benefício interessante a longo prazo, pois os projetos são seguros, tem um tempo de vida elevado (isso quer dizer que terá pouca ou nenhuma manutenção e um projeto durável), economia em contas (pois o projeto será baseado em seu consumo mensal então também não terá surpresas), investimento em uma energia limpa e renovável e uma valorização em seu empreendimento.

Uma pesquisa realizada pelo Laboratório Nacional Lawrence Berkeley, na Califórnia, descobriu que as pessoas estão dispostas a pagar até is mil dólares a mais por uma casa com placas solares instaladas.

Vale dizer que investir na energia do amanhã é um bom negócio pois ela traz inúmeros benefícios ao empreendimento, trata-se de uma energia natural e renovável, valoriza seu imóvel e se faz necessário atualmente. 


\section{OBJETIVO}

Uso da energia fotovoltaica para uso comercial, atuando em conjunto com a energia hidrelétrica para ter maior controle na conta de energia, contando a alta durabilidade dos painéis fotovoltaicos

\section{REVISÃO DE LITERATURA}

Brito (2018) assegura que entre todas as fontes de energias disponíveis, a energia solar é a mais abundante e pode ser explorada nas formas direta e indireta. A radiação solar transmitida na atmosfera terrestre sofre alterações ao entrar em contato com a superfície da Terra, devido as moléculas de hidrogênio existentes.

O Brasil possui um grande nível de insolação e reservas de quartzo com qualidade para a distribuição de energia solar. Segundo levantamentos realizados pela Agência Nacional de Energia Elétrica (ANEEL) e pela Associação Brasileira de Energia Solar Fotovoltaica (ABSOLAR), o Brasil alcançou a potência instalada de 4.46o MW em 2019, obtendo um crescimento de $45 \%$ em relação ao ano anterior, superando as expectativas.

Elon Musk apresentou a primeira telha solar da Tesla, em outubro de 2016, a tecnologia ganhou status e começou a atrair interessados. Segundo Elon Musk (2016), estimase que as novas telhas fotovoltaicas podem contar com uma vida útil de 50 anos.

\section{METODOLOGIA}

Segundo Martins et al (2004), ao atravessar a atmosfera, a radiação eletromagnética é atenuada por processos de espalhamento e absorção causados pelas partículas (denominados aerossóis) e moléculas de gases atmosféricos. Os coeficientes de absorção e de espalhamento dependem do comprimento de onda da radiação e das espécies de gases e aerossóis presentes na atmosfera.

A imagem a seguir demonstra os principais processos de interação da radiação solar e da radiação térmica na atmosfera. É um esquema simplificado do balanço radioativo do planeta, representando os processos do efeito médio global. Os valores dos componentes podem variar consideravelmente de acordo a região do planeta. 
Figura I - Diagrama simbólico dos processos de interação da radiação solar com a atmosfera terrestre.

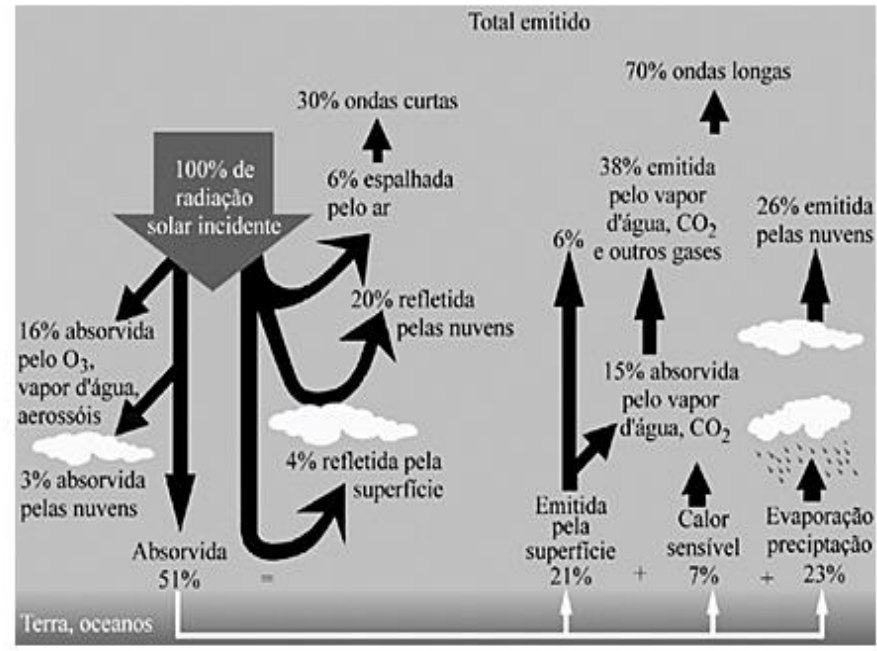

Figura 1 - Diagrama simbólico dos processos de interação da radiação solar com a atmosfera terrestre.

Fonte: (F.R. Martins, 2004)

A Agência Nacional de Energia Elétrica (Aneel) e a Associação Brasileira de Energia Solar Fotovoltaica (ABSOLAR) divulgaram um gráfico sobre a energia solar no Brasil com o ranking dos estados que utilizam essa fonte renovável de energia em todo o território brasileiro. O gráfico mostra que sistemas fotovoltaicos estão nas regiões Sudeste, Sul e Nordeste do Brasil, enquanto estados como Roraima e Amapá ficam atrás, com um baixo potencial de energia solar instalada.

Figura 2 - Energia Solar Fotovoltaica no Brasil.

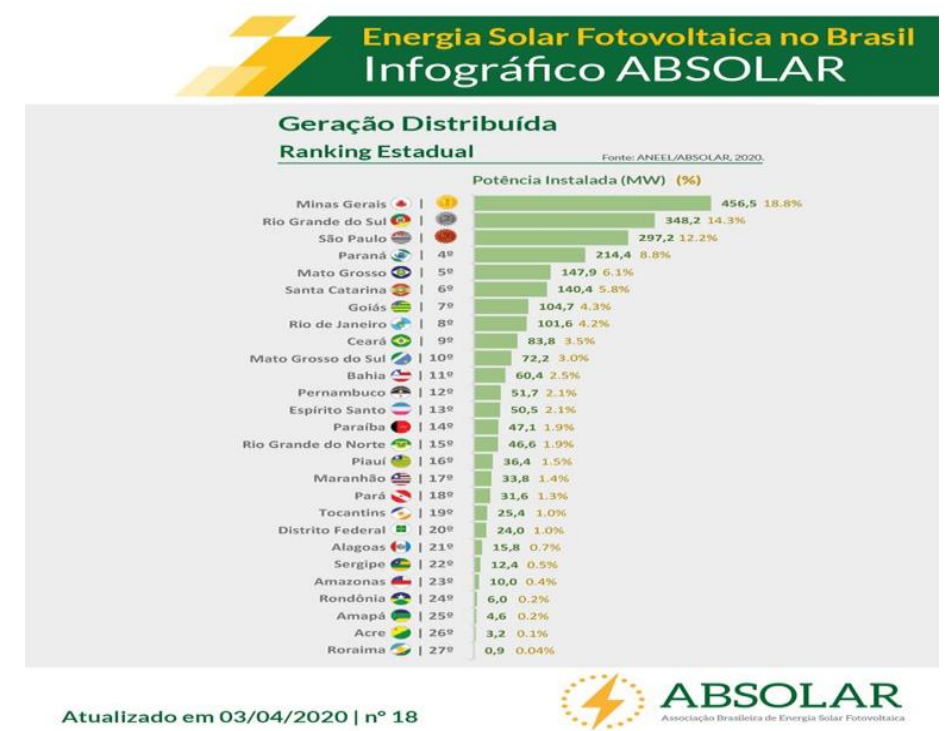

Fonte: (Aneel/ABSOLAR, 202I) 


\section{Resultados}

O potencial dos painéis fotovoltaicos vem sendo discutido incessantemente por se tratar de recursos tecnológicos que têm sido cada vez mais implantados em edificações que buscam unir eficiência e sustentabilidade. Juntamente a outras condutas de Green Building, a energia solar na construção civil é destaque como vantagem competitiva nesse setor.

O sistema de energia fotovoltaica tem a capacidade de abastecer totalmente a demanda elétrica de um empreendimento. Equipamentos conectados à rede, quando projetados corretamente, pagam apenas o custo de disponibilidade da distribuidora local.

\section{Figura 4 - Telhado com placas fotovoltaicas}

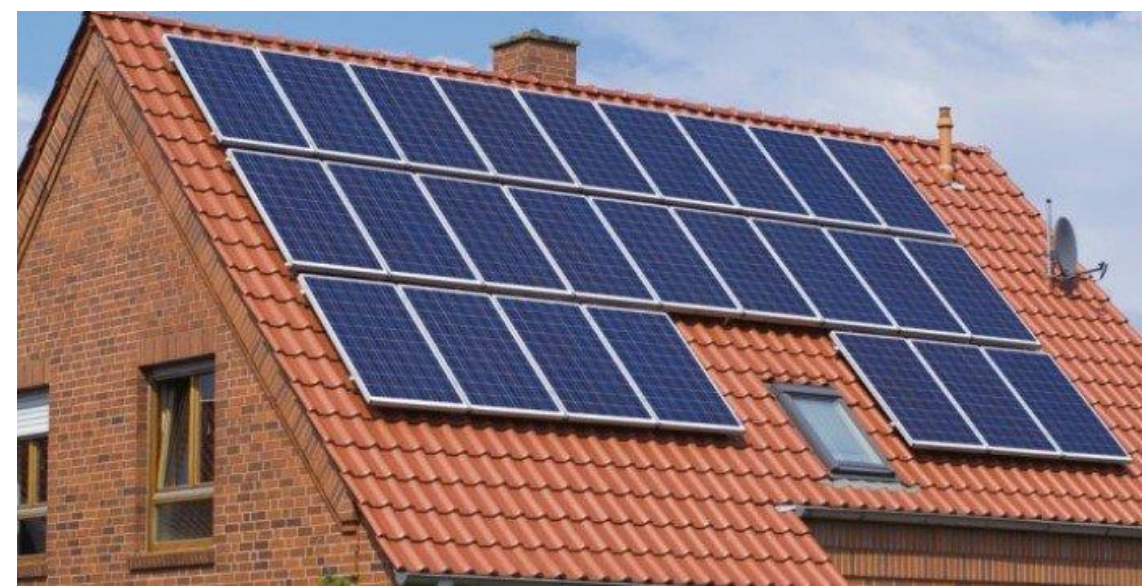

Telhado com placas fotovoltaicas (Boreal Solar, 2016).

\section{CONCLUSÃO}

Sistemas fotovoltaicos tendem a se tornar cada vez mais frequentes no Brasil, e garantir a qualidade desses sistemas deve ser uma preocupação constante. Nesse sentido, este trabalho apresenta um procedimento de qualificação de sistemas fotovoltaicos que deve ser utilizado com o objetivo de verificar seu correto funcionamento, a adequação às normas técnicas, a qualidade dos materiais, e comprovar (ou definir) sua capacidade de produção de energia.

Para atingir seu objetivo, o trabalho resumiu alguns aspectos relacionados às normas técnicas aplicáveis aos sistemas fotovoltaicos, apresentou os principais parâmetros de desempenho utilizados para caracterizar um sistema fotovoltaico, propôs uma metodologia para calcular a energia injetada na rede, a qual incorpora modelos novos e convencionais, e aplicou essa metodologia em um estudo de caso, que demonstrou sua confiabilidade.

Uma atividade importante de ser executada, e que serve como sugestão para trabalho futuro, é a aplicação completa do procedimento de qualificação em um sistema fotovoltaico conectado à rede. Por exemplo, em empresas de grande porte. 


\section{REFERÊNCIAS BIBLIOGRÁFICAS}

Agência Nacional de Energia Elétrica (Brasil). Atlas de energia elétrica do Brasil. Brasília: ANEEL, 2013.

Disponível: 〈http://www2.aneel.gov.br/aplicacoes/consulta_publica/documentos/NT_18oTA2 404.pdf ; Acesso em: mai. 2021.

ALMEIDA, P. M. Qualificação de sistemas fotovoltaicos conectados à rede, 2012. Disponível: $<$ https://teses.usp.br/teses/disponiveis/86/86131/tde-28062012102236/publico/DissertacaoMarceloPinhoAlmeida.pdf >; Acesso em: maio, 2021.

ALVES, D. E. J. A demanda de energia e o crescimento das fontes renováveis até 2035. 2017. Disponível: $\quad$ https://www.ecodebate.com.br/2017/o2/24/demanda-de-energia-e-ocrescimento-das-fontes-renovaveis-ate-2035-artigo-de-jose-eustaquio-diniz-alves/>; Acesso em: mai. 202I.

ALVES, G. E.; SILVA, F. A. Usando LEDs como fonte de energia, 2018. Disponível: 〈http://www.sbfisica.org.br/fne/Volg/NumI/led.pdf〉; Acesso em: mai. 2021.

BENEDITO, R. D. S. Caracterização da geração distribuída de eletricidade por meio de sistemas fotovoltaicos conectados à rede, no Brasil, sob os aspectos técnico, econômico e regulatório, 2009. Disponível: 〈https://teses.usp.br/teses/disponiveis/86/8613I/tde-I2082010I42848/publico/DissertRicardoBenedito.pdf>; Acesso em: mai. 202I.

BOQUIMPANI, L. C.; MOTTA, S. T. L. A.; SOUZA, C. L. O.; OlIVEIRA, R. V. H. C. Eficiência energética: Sistemas de iluminação com LEDs, distribuídos em corrente contínua e utilizando energia fotovoltaica. Disponível: 〈https://www.scielo.br/j/ac/a/b6xKHMSwZw6qWLTyfpfFgTs/?lang=pt〉; Acesso em: mai. 202I.

BRITO, M.E.; SILVA, R.O.; OLIVEIRA, A.M.; ESTENDER A.C.; FORMIGONI A.

Transição do Sistema Elétrico Tradicional para a Implantação do Sistema Fotovoltaico: $\begin{array}{llll}\text { Percepção } & \text { de } & \text { Funcionários, } & \text { Disponível: }\end{array}$ 〈https://revista.ufrr.br/adminrr/article/view/2727〉; Acesso em: mai. 202I. 
CAMARGO, F, ET AL. Desafios e oportunidades para energia solar fotovoltaica no Brasil. I edição, 2015 Disponível: 〈https://d3nehc6yl9qzo4.cloudfront.net/downloads/15_6_2015_wwf_energ_solar_final_web_3.p df $>$; Acesso em: mai. 2021.

CASTRO, M. G. RUI. Energias renováveis e produção descentralizada, 2022. Disponível: $<$ https://doczz.com.br/doc/37637/introdu\% $\mathrm{C}_{3} \% \mathrm{~A}_{7 \%} \mathrm{C}_{3} \% \mathrm{~A}_{30-\%} \mathrm{C}_{3} \%$ Ao-energiafotovoltaica-rui-m.g.-castro>; Acesso em: mai. 2021.

DEMONTI, R. Sistema de cogeração de energia a partir de painéis fotovoltaicos, I998. Disponível: 〈https://repositorio.ufsc.br/xmlui/handle/r23456789/77850〉; Acesso em: abr. 2021.

DIAS, L. S. Incorporação de Sistemas Fotovoltaicos em Envoltórias de edificações: Tecnologia e Arquitetura, 2014. Disponível: <http://dedalus.usp.br/F/74CI4328VAQK CJ ${ }_{4} \mathrm{C}_{4} \mathrm{BIK}_{3} \mathrm{QXR} 8 \mathrm{~L} 859 \mathrm{~J} 859 \mathrm{QXE} 2 \mathrm{QRFAE} 4 \mathrm{MYLVCCN66964}$ ?func=full- setset\&set_number= 005554\&se t_ entry=00ooor\&format=999>; Acesso em: mai. 2021.

IMHOFF, J. Desenvolvimento de conversores estáticos para sistemas fotovoltaicos autônomos,

Disponível: 〈https://repositorio.ufsm.br/bitstream/handle/I/86o8/JOHNINSONIMHOFF.pdf〉; Acesso em: abr. 2021.

MARTINS, ET AL. Levantamento dos recursos de energia solar no Brasil com o emprego de satélite geoestacionário - o Projeto Swera, 2004. Disponível: 〈https://www.scielo.br/j/rbef/a/vr68FHKFh6PWkYwtYXYvfMj/?format=pdf\&lang=pt〉; Acesso em: mai. 2021.

NAKAGAMI, H.; TANAKA, A.; MURAKOSHI, C.; LITT, B. Stand-by Electricy $\begin{array}{llll}\text { consumption } & \text { Japanese } & \text { houses, D99. Disponível: }\end{array}$ 〈https://link.springer.com/chapter/Io.1007/978-3-642-60020-3_42〉; Acesso em: mai. 202I. PEREIRA, E.; MARTINS, F.; ABREU, S.; RÜTHER, R. Atlas brasileiro de energia solar. I $\mathrm{a}^{\mathbf{a}}$ edição, 2006.

Disponível: $<$ https:// 
https://energypedia.info/images/9/95/Brazilian_Atlas_of_Solar_Energy.pdf $>$; Acesso em: abr. 2021.

PRIEB, C. Desenvolvimento de um sistema de ensaio de módulos fotovoltaicos, 2002 Disponível: 〈https://lume.ufrgs.br/handle/ror83/209I〉; Acesso em: abr. 202I.

PRIEB, M. W. C. Desenvolvimento de um sistema de ensaio de módulos fotovoltaicos, 2002. Disponível:

$<$ https://lume.ufrgs.br/bitstream/handle/roI83/2091/oo0363966.pdf? sequence=I\&isAllowed =y >; Acesso em: mai, 2021.

THIRUGNANASAMBANDAM, ET AL. A review of solar thermal technologies, 2010. Disponível: 〈https://www.sciencedirect.com/science/article/abs/pii/Si364032109001750〉; Acesso em: mai, 2021. 